8. The second paper by Professor Gutzmer consists of a short report on some investigations and results related to a previous paper by him under the title: "Theory of adjoined differential equations," Halle, 1896.

The next meeting of the Vereinigung will be held at Meran, in the Tyrol, in September, 1905, under the presidency of Professor F. Klein.

Göttingen, Germany,

R. E. WIILSON.

November, 1904.

\title{
THE CONSTRUCTION OF CONICS UNDER GIVEN CONDITIONS.
}

BY PROFESSOR M. W. HASKELL.

(Read before the San Francisco Section of the American Mathematical Society, April 30, 1904.)

UNDER the above title are grouped a few notes on special aspects of the subject, with no pretense at an exhaustive treatment. While certain of the results are not new, it is believed that the method by which they are derived will be of some interest. Homogeneous coördinates are used exclusively ; following Casey (Analytical Geometry, page 70), a quadrangle or quadrilateral whose diagonal triangle is the triangle of reference is designated as a "standard" quadrangle or quadrilateral. The coördinates of the vertices of a standard quadrangle, or of the sides of a standard quadrilateral, are then $\pm \kappa_{1}: \pm \kappa_{2}: \pm \kappa_{3}$, and they may be projected into $\pm 1: \pm 1: \pm 1$ if desired. It is to be noticed that the complete quadrangle (quadrilateral) is fully determined and can be easily constructed, when the diagonal triangle and any one vertex (side) are given.

§1. Conics Defined by Five Points or by Five Tangents.

Theorem I. The eight vertices of two standard quadrangles lie on one and the same conic.

For, let the vertices be respectively $\pm \kappa_{1}: \pm \kappa_{2}: \pm \kappa_{3}$ and $\pm \lambda_{1}: \pm \lambda_{2}: \pm \lambda_{3}$; it is clear that they all lie on the conic whose equation is 


$$
\left|\begin{array}{rrr}
x_{1}^{2} & x_{2}^{2} & x_{3}^{2} \\
\kappa_{1}^{2} & \kappa_{2}^{2} & \kappa_{3}^{2} \\
\lambda_{1}^{2} & \lambda_{2}^{2} & \lambda_{3}^{2}
\end{array}\right|=0 .
$$

This theorem affords the following simple construction by points of a conic of which five points are given.

Constructron : Construct the complete quadrangle of any four of the given points, and regard its diagonal triangle as the triangle of reference. Then complete the standard quadrangle of which the fifth given point is one vertex. The remaining three vertices lie on the conic. The five given points lead us thus immediately to fifteen new points, and the construction can be carried on indefinitely.

I will omit here, and in general in what follows, all theorems which can be derived from those given merely by the principle of duality.

Theorem II (Lemma). The lines joining corresponding vertices of two standard quadrangles form a standard quadrilateral.

For, if the vertices of the given quadrangles be respectively $\pm \kappa_{1}: \pm \kappa_{2}: \pm \kappa_{3}$ and $\pm \lambda_{1}: \pm \lambda_{2}: \pm \lambda_{3}$, the coördinates of the lines joining corresponding vertices will be

$$
\left.\pm\left(\kappa_{2} \lambda_{3}-\kappa_{3} \lambda_{2}\right): \pm\left(\kappa_{3} \lambda_{1}-\kappa_{1} \lambda_{3}\right): \pm \kappa_{1} \lambda_{2}-\kappa_{2} \lambda_{1}\right) .
$$

Consider now two standard quadrangles $A B C D, A^{\prime} B^{\prime} C^{\prime} D^{\prime}$ and the conic upon which they lie. If $A^{\prime}$ be made to approach $A, B^{\prime} C^{\prime} D^{\prime}$ will approach $B C D$ respectively, the chords $A A^{\prime}$, $B B^{\prime}, C C^{\prime}, D D^{\prime}$ approaching the tangents at $A, B, C, D$. Hence

Theorem III. The tangents at any four points of a conic form a quadrilateral which is standard with reference to the diagonal triangle of the quadrangle of those four points.

From this theorem and from the dual of Theorem I we have immediately

Theorem IV. Let the four intersections of two conics form a standard quadrangle. Then the two quadrilaterals formed by their tangents are standard, and therefore envelop a conic.

Similarly, the eight points of contact of the four common tangents to two conics lie on a conic. 
Theorem V. The quadrilateral of the four common tangents to two conics has the same diagonal triangle as the quadrangle of their intersections.

For, let $P P^{\prime}$ be one of these common tangents, $P$ and $P^{\prime}$ being the respective points of contact. Then, if $Q, R, S$; $Q^{\prime}, R^{\prime}, S^{\prime}$, respectively be the remaining vertices of the quadrangles determined by $P$ and by $P^{\prime}, Q Q^{\prime}, R R^{\prime}$ and $S S^{\prime}$ will be the remaining sides of the quadrilateral determined by $P P^{\prime}$. $Q, R, S$ will lie on the first conic; $Q^{\prime}, R^{\prime}, S^{\prime}$ on the second; and $Q Q^{\prime}, R R^{\prime}, S S^{\prime}$ will be tangent to both.

Moreover, the conic through the eight points of contact of the common tangents, and the conic touched by the eight tangents at the intersections of two conics, each have the triangle of reference as a self-polar triangle.

§2. Harmonic Ranges on a Conic. Conics through Four given Points and Tangent to a given Line.

Theonem VI.-Let $A_{1}, A_{2}, A_{3}, A_{4}, P$ be five points on a conic. Let $A_{i k}$ be the harmonic conjugate of $P$ with respect to $A_{i}$ and $A_{k}$. Then the lines joining $A_{12}$ and $A_{34}, A_{13}$ and $A_{24}$, $A_{14}$ and $A_{23}$ are concurrent with the tangent at $P$.

Let the coördinates of the five points be

$$
\begin{gathered}
A_{1}(1: 1: 1), A_{2}(-1: 1: 1), A_{3}(1:-1: 1), A_{4}(1: 1:-1), \\
P(\lambda: \mu: \nu) .
\end{gathered}
$$

It is not difficult to show that the equations of the three lines in question are

$$
\begin{aligned}
& A_{12} A_{34} \lambda\left(-2 \lambda^{2}+\mu^{2}+\nu^{2}\right) x_{1}-\mu\left(\nu^{2}-\lambda^{2}\right) x_{2}+\nu\left(\lambda^{2}-\mu^{2}\right) x_{3}=0 \\
& A_{13} A_{24} \lambda\left(\mu^{2}-\nu^{2}\right) x_{1}+\mu\left(\lambda^{2}-2 \mu^{2}+\nu^{2}\right) x_{2}-\nu\left(\lambda^{2}-\mu^{2}\right) x_{3}=0 \\
& A_{14} A_{23}-\lambda\left(\mu^{2}-\nu^{2}\right) x_{1}+\mu\left(\nu^{2}-\lambda^{2}\right) x_{2}+\nu\left(\lambda^{2}+\mu^{2}-2 \nu^{2}\right) x_{3}=0
\end{aligned}
$$

and these lines evidently meet in

$$
Q \quad\left(\frac{1}{\lambda}: \frac{1}{\mu}: \frac{1}{\nu}\right)
$$

Moreover, the equation of the tangent at $P$ is

$$
\lambda\left(\mu^{2}-\nu^{2}\right) x_{1}+\mu\left(\nu^{2}-\lambda^{2}\right) x_{2}+\nu\left(\lambda^{2}-\mu^{2}\right) x_{3}=0,
$$

and this is satisfied by the coördinates of $Q$. 
Now the points $P$ and $Q$ are interchanged by the involutory quadratic transformation

$$
y_{1}: y_{2}: y_{3}=\frac{1}{x_{1}}: \frac{1}{x_{2}}: \frac{1}{x_{3}},
$$

of which the triangle of reference is the fundamental triangle and the points $A_{1}, A_{2}, A_{3}, A_{4}$ are the double points. Hence the line $P Q$ is also tangent to the conic through $A_{1}, A_{2}, A_{3}$, $A_{4}$ and $Q$, and we are led to the following interpretation of the above transformation :

THEOREM VII. The pairs of points which are interchanged by an involutory quadratic transformation of the plane are the points of contact of the tangents common to the pairs of conics of the pencil through the four double points of the transformation.

Or again, they are the double points of the involutions defined on the lines of the plane by the pencil of conics through four points.

In general, under this transformation a straight line is converted into a conic through the vertices of the fundamental triangle; but this conic degenerates if the line in question passes through one of the vertices of the fundamental triangle, and in particular the sides of the complete quadrangle of the self-corresponding points are converted into themselves, corresponding point-pairs on one of these sides being harmonically conjugate with reference to the self-corresponding points of that side. For instance, the points $(1+\lambda: 1-\lambda: 1+\lambda)$ and $(1-\lambda$ : $1+\lambda: 1-\lambda)$ are evidently interchanged by the transformation, and they are harmonic conjugates with respect to $(1: 1: 1)$ and $(1:-1: 1)$. Hence follows

Theorem VIII. The conic into which a given line is converted by the quadratic transformation passes through the harmonic conjugates of the intersections of that line with the six sides of the complete quadrangle $A_{1} A_{2} A_{3} A_{4}$.

A special case of this theorem is the conic passing through the middle points of the sides of a complete quadrangle and through the vertices of its diagonal triangle. It can also easily be shown that the lines joining the pairs of such points which lie on opposite sides of the quadrangle are concurrent.

These theorems lead us immediately to the construction of the two conics through four given points and tangent to a given line. For the respective points of contact are the intersections of the given line with the conic into which it is converted by 
the quadratic transformation. They are also of course the double points of the involution defined upon that line by its intersection with the three pairs of opposite sides of the complete quadrangle of the four given points.

The question of the reality of the conics can be determined readily from the above. For the line $a x_{1}+b x_{2}+c x_{3}=0$ will intersect the conic $a x_{2} x_{3}+b x_{3} x_{1}+c x_{1} x_{2}=0$ in real points if

$$
s(s-a)(s-b)(s-c)<0
$$

where $2 s=a+b+c$, and the geometric interpretation of this result is that the given line must cut each pair of opposite sides of the complete quadrangle of the four points either in the segments upon which a vertex of the diagonal triangle lies, or else in those segments upon which it does not lie. If this condition is fulfilled for one pair of sides, it is also fulfilled for both the remaining pairs.

It may be worth while to remark here that the quadratic transformation is fully defined by the fundamental triangle and any pair of corresponding points $P_{1}$ and $Q_{1}$, the double points being determined by completing the standard quadrangles $P_{1} P_{2} P_{3} P_{4}$ and $Q_{1} Q_{2} Q_{3} Q_{4}$ and passing a conic through each of these sets of points and tangent to $P_{1} Q_{1}$; for the intersections of these two conics will be the double points required. This construction solves the problem of finding two conics, given their common self-polar triangle and the points of contact of one of their common tangents.

\section{§3. Conics with Tuo Points and Three Tangents given.}

Let the three tangents be taken as the triangle of reference and let the two points be $\left(a_{1}: b_{1}: c_{1}\right)$ and $\left(a_{2}: b_{2}: c_{2}\right)$. Then, writing

$$
\begin{aligned}
l & =\left(\sqrt{b_{1} c_{2}}-\sqrt{b_{2} c_{1}}\right)^{2}, & l^{\prime} & =\left(\sqrt{b_{1} c_{2}}+\sqrt{b_{2} c_{1}}\right)^{2}, \\
m & =\left(\sqrt{c_{1} a_{2}}-\sqrt{c_{2} a_{1}}\right)^{2}, & m^{\prime} & =\left(\sqrt{c_{1} a_{2}}+\sqrt{c_{2} a_{1}}\right)^{2}, \\
n & =\left(\sqrt{a_{1} b_{2}}-\sqrt{a_{2} b_{1}}\right)^{2}, & n^{\prime} & =\left(\sqrt{a_{1} b_{2}}+\sqrt{a_{2} b_{1}}\right)^{2},
\end{aligned}
$$

the equations of the four conics satisfying the given conditions will be

1) $\quad l u_{2} u_{3}+m u_{3} u_{1}+n u_{1} u_{2}=0$,

2) $l u_{2} u_{3}+m^{\prime} u_{3} u_{1}+n^{\prime} u_{1} u_{2}=0$, 
3) $\quad l^{\prime} u_{2} u_{3}+m u_{3} u_{1}+n^{\prime} u_{1} u_{2}=0$,

4) $l^{\prime} u_{2} u_{3}+m^{\prime} u_{3} u_{1}+n u_{1} u_{2}=0$.

If the given points and lines are all real, then these conics are all real if no two of the given lines meet the line joining the two given points in points alternating with the given points; otherwise they are all imaginary.

Each pair of these conics will have a fourth common tangent, for example

$$
\begin{array}{ll}
t_{12}: & \frac{x_{1}}{m n^{\prime}-m^{\prime} n}+\frac{x_{2}}{l\left(n-n^{\prime}\right)}-\frac{x_{3}}{l\left(m-m^{\prime}\right)}=0, \\
t_{34}: & \frac{x_{1}}{m^{\prime} n^{\prime}-m n}+\frac{x_{2}}{l^{\prime}\left(n-n^{\prime}\right)}+\frac{x_{3}}{l\left(m-m^{\prime}\right)}=0,
\end{array}
$$

and it can easily be shown that the intersections of opposite pairs $t_{12}$ and $t_{34}, t_{13}$ and $t_{24}, t_{14}$ and $t_{23}$ all lie on the line joining the two given points.

The poles of the common chord. The four conics are fully defined as soon as the poles of the common chord are determined. Now, we have identically

$$
\begin{gathered}
l u_{2} u_{3}+m u_{3} u_{1}+n u_{1} u_{2} \\
\equiv\left(a_{1} u_{1}+b_{1} u_{2}+c_{1} u_{3}\right)\left(a_{2} u_{1}+b_{2} u_{2}+c_{2} u_{3}\right) \\
-\left(u_{1} \sqrt{a_{1} a_{2}}+u_{2} \sqrt{b_{1} b_{2}}+u_{3} \sqrt{c_{1} c_{2}}\right)^{2},
\end{gathered}
$$

and similarly for the other conics. Hence the four poles in question are the points

$$
\sqrt{a_{1} a_{2}}: \pm \sqrt{b_{1} b_{2}}: \pm \sqrt{c_{1} c_{2}} .
$$

These points form a standard quadrangle and they are the double points of the quadratic transformation

$$
y_{1}: y_{2}: y_{3}=\frac{a_{1} a_{2}}{x_{1}}: \frac{b_{1} b_{2}}{x_{2}}: \frac{c_{1} c_{2}}{x_{3}},
$$

which interchanges the two given points, the three given lines being the fundamental triangle.

University of CaLIFornia, April, 1904. 